\section{Seborrheic keratosis evolution into squamous cell carcinoma: A truly modified sun-related tumor? A case report and review of the literature}

\author{
Ioana Cimpean, ${ }^{1}$ Ivan Theate, ${ }^{2}$ \\ Olivier Vanhooteghem ${ }^{1}$
}

${ }^{1}$ Department of Dermatology, Saint

Elisabeth Hospital, CHU UCL Namur;

${ }^{2}$ Department of Pathology, IPG

Gosselies, Belgium

\begin{abstract}
The incidence of seborrheic keratosis (SK) generally increases with age and are mostly localized on the trunk, face and neck, especially on sun-exposed areas. The association between SK and skin malignancies appears to be accidental, but in situ transformation occurs more frequently in sun-exposed areas. Histopathological examination of all SK cases should be considered, especially when SK lesions exhibit atypical clinical manifestations, such as ulceration and cresting, as they may herald malignant transformation. In addition, other features associated with malignant transformation include excoriations or hemorrhages identified on the lesion, modification and evolution of the macroscopic characteristics, and the presence of local erythema or pruritus. Immunocompromised patients exhibit an increased risk of malignant transformation, even when radiation is involved.
\end{abstract}

\section{Introduction}

The incidence of seborrheic keratosis (SK) generally increases with age. These lesions are mostly localized on the trunk, face and neck, especially on sun-exposed areas. Basal cell carcinoma (BCC), squamous cell carcinoma (SCC) and melanoma associated with SK may arise from the basaloid cells, squamous cells and melanocytes that are contained in SK. Here, we report the in situ transformation of SK into SCC that was unfortunately treated by local cryotherapy a few months prior to surgical treatment.

\section{Case Report}

A 69-year-old male who worked out- doors and lacked medical history, with the exception of heliodermatitis, presented to our dermatological department for an SK lesion localized on the right flank. The lesion appeared few years ago but became painful in recent months, exhibiting peripheral inflammation and superficial bleeding (Figure 1). The lesion was treated by local cryotherapy, but the lesion recurred. Given the clinical appearance of the lesion, we performed surgical treatment because SCC was suspected and confirmed by histopathology analysis (Figures 2 and 3). Follow-up (7 years) showed no tumor recurrence.

\section{Discussion}

The incidence of SK typically increases with age, and most lesions occur on the trunk, face and neck. The lesion size can vary from $0.5 \mathrm{~cm}$ to $3.0 \mathrm{~cm}$ or larger in diameter. The etiology is misunderstood. Lesions appear to be genetically stable despite multiple somatic alterations. ${ }^{1}$ Increased FGFR3 mutation levels are associated with increased FOXN1 levels, and a positive feedback loop is hypothesized to favorise malignant progression in UV-prone areas. ${ }^{2}$ Histologically, hyperkeratosis, papillomatosis or acanthosis are features observed in SK, and these lesions tend to be reticulated, pigmented, clonal, irritated or flat. Clinically, keratinocyte proliferation can lead to the formation of an endophytic or exophytic nodule. The lesion typically exhibits a well-defined border with the underlying dermis. Some lesions may be symptomatic due to pruritus and are often treated by local cryotherapy. Multiple lesions are occasionally observed in cases with an internal neoplasm called a LeserTrelat sign, which is somtimes present in gastrointestinal tract adenocarcinoma; breast, lung, liver, pancreas, and prostate cancers; hematopoietic diseases ${ }^{3}$ and metastasized melanoma. ${ }^{4}$ A pseudo Leser-Trelat sign is associated with chemotherapy and epidermal growth factor antagonists therapy. ${ }^{5}$ Non-malignant cases with LeserTrelat signs are rarely described. Malignant transformation raises questions about the true nature of these benign lesions. The malignant transformation of SK appears more frequently in the elderly on the head and neck. Malignant lesions are occasionally associated with a recent history of pruritus, ulceration or increasing size. Potential etiological factors include prolonged sun damage and chronic low-dose radiation exposure. ${ }^{6}$ In a study involving more than 23000 histopathological examinations, $11.9 \%$ of clinically apparent of SK lesions
Correspondence: Olivier Vanhooteghem, Department of Dermatology, Saint Elisabeth Hospital, Place Louise Godin, B-5000 Namur, Belgium.

E-mail: ovanhooteghem@hotmail.com

Key words: Seborrheic keratosis; Squamous cell carcinoma; Sun.

Contributions: the authors contributed equally.

Conflict of interest: the authors declare no potential conflict of interest.

Funding: none.

Received for publication: 26 January 2019.

Revision received: 14 March 2019.

Accepted for publication: 14 March 2019.

This work is licensed under a Creative Commons Attribution-NonCommercial 4.0 International License (CC BY-NC 4.0).

CCopyright I. Cimpean et al., 2019

Licensee PAGEPress, Italy

Dermatology Reports 2019; 11:7999

doi:10.4081/dr.2019.7999

were diagnosed as BCC, $3.4 \%$ as SCC and $1.01 \%$ as melanoma. ${ }^{7}$ In another retrospective study of 813 SK lesions, neoplasia was noted in 5.3\%; superficial SCC was the most common, followed by $\mathrm{BCC}$ and invasive SCC. Most lesions may represent SK an in situ transformation. ${ }^{8}$ On the other hand, a review of greater than 10000 SK revealed 14 cases of BCC; however, the majority of these lesions were in direct contact with the epidermis ${ }^{9}$ as presented by Ishida who suggested that BCC does not arise directly from SK but that SK is distinct from the carcinoma based on immunohistochemical results. ${ }^{10}$ The presence of a melanoma inside of an SK lesion prompts the question - does this lesion represent a coincidental association or true malignant transformation? Given that this association is very rare, it seems to be coincidental. However, dermoscopic analysis of any suspicious or changing SK lesion is essential. ${ }^{11}$ In a study of 9204 cases, melanoma was identified in $0.66 \%$ of cases submitted for histological examination with a clinical diagnosis that included SK. Melanoma was in the clinical differential diagnosis of $51 \%$ lesions. Of the remaining lesions, $28 \%$ had a differential diagnosis of SK vs melanocytic nevus, $12 \%$ BCC, or $5 \%$ squamous cell proliferation. All the histological types of melanoma were described. ${ }^{12}$ Although rare, the association SK and eccrine porocarcinoma has been described. ${ }^{13}$ The molecular mechanism of a malignant transformation is not completely understood. Proposed theories implicate the alter- 


\section{Case Report}

ation of proteins involved in cell cycle regulation. In general, epidermal growth factor receptors (EGFR) are present on basal keratinocytes, but EGFR levels decrease as the keratinocytes differentiate in the upper epidermal layers. The loss of the membrane receptor with the absence of cytoplasmic EGFR, which is similar to actinic keratosis, or cytoplasmic receptor accumulation, which is similar to Bowen's disease, has been observed. ${ }^{14}$ Somatic fibroblast growth factor receptor 3 (FGFR3) and phosphatidylinositol 3-kinase catalytic subunit $\alpha$ (PIK3CA) mutations are found in $89 \%$ of SK lesions and may be important in the development of cutaneous epithelial malignancies, ${ }^{15}$ especially those associated with increased age and localization on sunexposed areas of the head and neck. ${ }^{16}$ Dupperet et al. suggested FGFR3 activation in skin keratinocytes might play a tumorsuppressive role by driving differentiation and antagonizing RAS signaling. These authors postulated that activating FGFR3 mutations alone are insufficient to drive KS and do not alter the growth kinetics or differentiation status of SCC and that FGFR3 pro- tein itself is dispensable for Ras-driven SCC. ${ }^{17}$ The expression of glioma tumor-suppressor candidate region gene 2 (GLTSCR2) regulates the stability of the tumor suppressor PTEN, and the tumor suppressive function of GLTSCR2 is reduced in SK. ${ }^{18}$

\section{Conclusions}

In general, the association between SK and skin malignancies appears to be accidental, but in situ transformation occurs

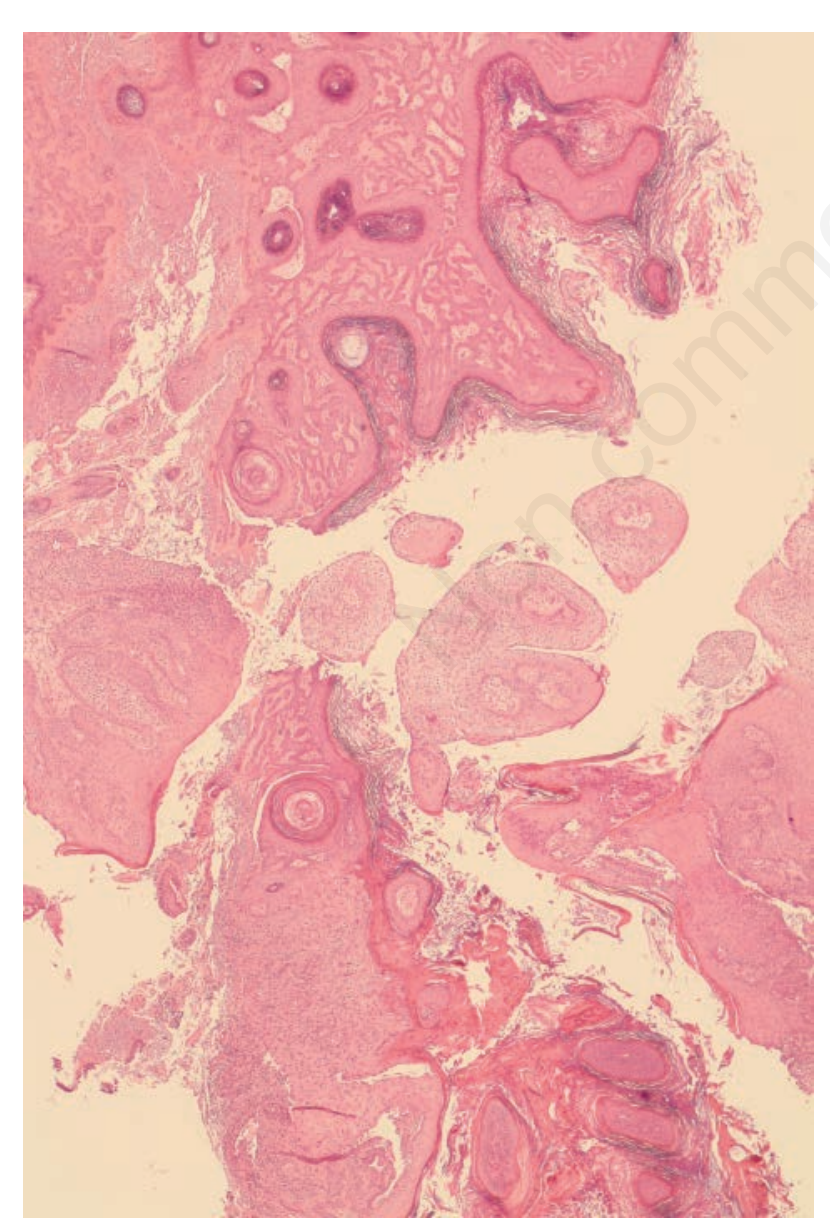

Figure 2. Papillomatous seborrheic keratosis with massive hyperkeratosis and papillomatosis (Haematoxylin Eosin magnification $\times 2.5)$.

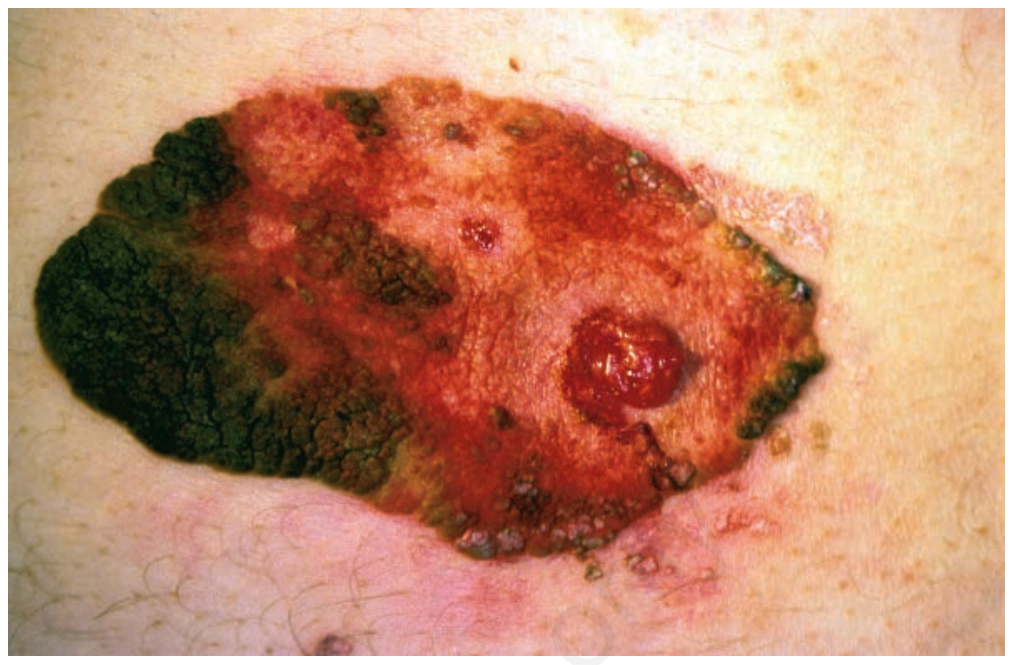

Figure 1. Painful seborheic keratosis with peripheral inflammation and superficial bleeding on the half right part.

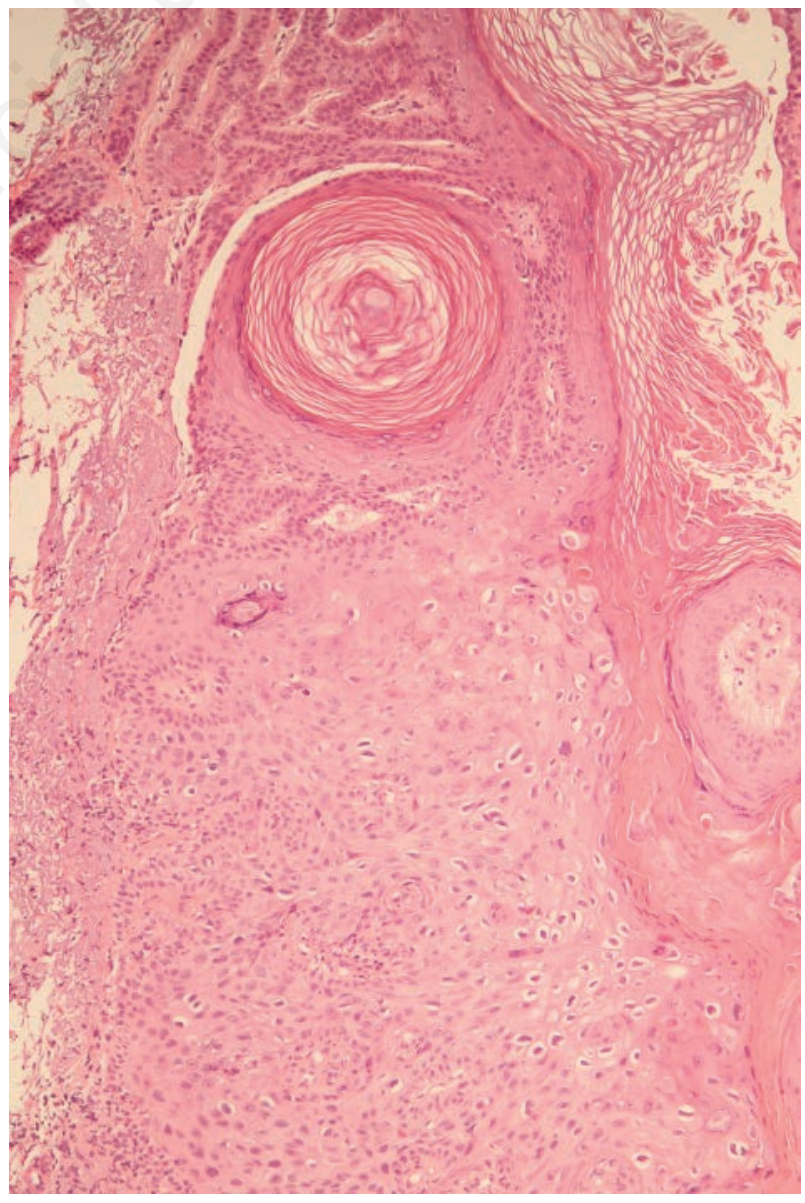

Figure 3. Foci of transformation with atypical nuclei and anisokaryosis (Haematoxylin Eosin magnification $\times 10$ ). 
more frequently in sun-exposed areas. ${ }^{6}$ Histopathological examination of all SK cases should be considered, especially when SK lesions exhibit atypical clinical manifestations, such as ulceration and cresting, as they may herald malignant transformation. In addition, other features associated with malignant transformation include excoriations or hemorrhages identified on the lesion, modification and evolution of the macroscopic characteristics, and the presence of local erythema or pruritus. Immunocompromised patients exhibit an increased risk of malignant transformation, ${ }^{19}$ even when radiation is involved. ${ }^{20}$

\section{References}

1. Hafner C, Toll A, Fernandez-Casado A, et al. Multiple oncologenic mutations and clonal relationship in spatially distinct benign human epidermal tumors. Proc Natl Acad Sci USA 2010;107: 20780-5.

2. Heidenreich B, Denisova E, Rachakonda S, Sanmartin O. Genetic alterations in seborrheic keratoses. Oncotarget 2017;8:36639-49.

3. Narala S, Cohen PR. Cutaneous T-cell lymphoma-associated Leser-Trélat sign: report and world literature review. Dermatol Online J 2017;1:15-23.

4. Siedek V, Schuh T, Wollenberg A. Leser-Trelat sign in metastasized malignant melanoma. Eur Arch Otorhinolaryngol 2009;266:297-9.

5. Lilly E, Granter SR, Haynes HA, Ibrahimi OA. Chemotherapy-induced inflammatory seborrheic keratoses in a man with acute myeloid leukemia: a variant of Leser-Trélat sign? Cutis 2012;90:235-6.

6. Gaffney DC, Muir JB, De'Ambros B. Malignant change in seborrhoeic keratoses in a region with high solar ultraviolet levels. Australas J Dermatol 2014;55:142-4.

7. Rigopoulos D, Rallis E, ToumbisIoannou E, et al. Seborrhoeic keratosis or occult malignant neoplasm of the skin? J Eur Acad Dermatol Venereol 2002;16:168-70.

8. Vun Y, De'Ambrosis B, Spelman L, et al. Seborrhoeic keratosis and malignancy: collision tumour or malignant transformation? Australas J Dermatol 2006; 47:106-8.

9. Mikhail GR, Mehregan AH. Basal cell carcinoma in seborrheic keratosis. J Am Acad Dermatol 1982;6:500-6.

10. Ishida M, Ohsato N, Okabe H. Basal cell carcinoma arising within a seborrheic keratosis with respect to immunohistochemical characteristics. Oncol Lett 2011;2:625-7.

11. Braga JC, Scope A, Klaz I, Mecca P, et al. Melanoma mimicking seborrheic keratosis: an error of perception precluding correct dermoscopic diagnosis. J Am Acad Dermatol 2008;58:875-80.

12. Izikson L, Sober AJ, Mihm MC Jr, Zembowicz A. Prevalence of melanoma clinically resembling seborrheic keratosis: analysis of 9204 cases. Arch Dermatol 2002;138:1562-6.

13. Hoshina D, Akiyama M, Hata $\mathrm{H}$, et al. Eccrine porocarcinoma and Bowen's disease arising in a seborrhoeic kerato- sis. Clin Exp Dermatol 2007;32:54-6.

14. Groves RW, Allen MH, MacDonald DM. Abnormal expression of epidermal growth factor receptor in cutaneous epithelial tumours. J Cutan Pathol 1992; 19:66-72.

15. Logié A, Dunois-Lardé C, Rosty C, et al. Activating mutations of the tyrosine kinase receptor FGFR3 are associated with benign skin tumors in mice and humans. Hum Mol Genet 2005;14: 1153-60.

16. Hafner C, Hartmann A, van Oers JM, et al. FGFR3 mutations in seborrheic keratoses are already present in flat lesions and associated with age and localization. Mod Pathol 2007;20:895-903.

17. Duperret EK, Oh SJ, McNeal A, et al. Activating FGFR3 mutations cause mild hyperplasia in human skin, but are insufficient to drive benign or malignant skin tumors. Cell Cycle 2014;13: 1551-9.

18. Kim JY, Kim HS, Lee S, Park JH. The expression of GLTSCR2, a candidate tumor suppressor, is reduced in seborrheic keratosis compared to normal skin. Pathol Res Pract 2010;206:295-9.

19. Conic RZ, Napekoski K, Schuetz H, et al. The role of immunosuppression in squamous cell carcinomas arising in seborrheic keratosis. J Am Acad Dermatol 2017;76:1146-50.

20. Suvarna SK, Bagary M, Glazer G. Radiation-induced squamous carcinoma arising within a seborrhoeic keratosis. Br J Dermatol 1993;128:443-7. 